\title{
Is intra-abdominal hypertension a risk factor for ventilator-associated pneumonia?
}

\author{
Eleni Papakrivou, Eustratios Manoulakas, Epaminondas Zakynthinos, Demosthenes Makris \\ Department of Critical Care Medicine, University Hospital of Larisa, University of Thessaly School of Medicine, Larisa, Greece \\ Contributions: (I) Conception and design: E Papakrivou, D Makris; (II) Administrative support: E Zakynthinos; (III) Provision of study materials \\ or patients: E Papakrivou, D Makris; (IV) Collection and assembly of data: E Papakrivou, E Manoulakas; (V) Data analysis and interpretation: \\ E Papakrivou, D Makris; (VI) Manuscript writing: All authors; (VII) Final approval of manuscript: All authors. \\ Correspondence to: Demosthenes Makris, MD, PhD. Department of Critical Care Medicine, University Hospital of Larisa, University of Thessaly \\ School of Medicine, Biopolis, Larisa 41110, Greece. Email: appollon7@hotmail.com.
}

\begin{abstract}
In the last years, there has been a significant amount of research about the impact of intraabdominal hypertension (IAH) on the outcomes of critical care patients. IAH is increasingly recognized as potential complication in intensive care unit (ICU) patients. IAH affects all body systems, most notably the cardiac, respiratory, renal, and neurologic systems. IAH affects blood flow to various organs and plays a significant role in the prognosis of the patients. Recognition of IAH, its risk factors and clinical signs can reduce the morbidity and mortality associated. Moreover, knowledge of the pathophysiology may help rationalize the therapeutic approach. On the other hand, ICU patients present frequently ventilatorassociated respiratory infections. Ventilator-associated pneumonia (VAP) is the most common healthcareassociated infection (HAI) in adult critical care units. It is associated with increased ICU stay, patient ventilator days and mortality. This paper reviews the relationship between IAH and VAP. Despite animal experimentation and physiological studies on humans, in favor of the impact of IAH to VAP, there is no definitive clinical data that IAH is associated with VAP. Microaspirations form the gastrointestinal track is a pathophysiological mechanism for VAP. This review provides data suggesting that under IAH conditions bacterial translocation might be an additional responsible mechanism for VAP in those patients that merits further investigation in the future.
\end{abstract}

Keywords: Bacteremia; bacterial translocation; intra-abdominal hypertension (IAH); intra-abdominal pressure (IAP); sepsis; ventilator-associated pneumonia (VAP)

Submitted Jun 29, 2018. Accepted for publication Jul 19, 2018.

doi: 10.21037/atm.2018.08.27

View this article at: http://dx.doi.org/10.21037/atm.2018.08.27

\section{Introduction}

Ventilator-associated pneumonia (VAP) is associated with considerable morbidity (1) while some studies have associated VAP with mortality (2). Previous studies have identified several risk factors for VAP. It has been also suggested that the risk for VAP might be increased in cases with intra-abdominal hypertension (IAH) (3). Animal experimental and human physiologic studies suggested that IAH can cause impairment of respiratory function (4-6). IAH has a serious impact on the function of respiratory as well as peripheral organs. In the presence of alveolar capillary damage, which occurs in acute respiratory distress syndrome (ARDS), IAH promotes lung injury as well as edema, impedes the pulmonary lymphatic drainage, and increases intra-thoracic pressures, leading to atelectasis, airway closure, and deterioration of respiratory mechanics and gas exchange which in turn, may increase the risk for infection (3). On the other hand, a main pathophysiological mechanism for pneumonia in critical care patients is the aspiration of contaminated with pathogenic organisms' gastric secretions (7) that could be aggravated by increased 
intra-abdominal pressure (IAP) (8). Furthermore, it cannot be excluded that bacterial translocation via the bloodstream in the lungs could be an additional mechanism for pneumonia during IAH (9).

In this article we reviewed the available literature to study whether increased IAP is a risk factor for increased VAP frequency.

\section{Methods}

Data for this review were identified through searches of PubMed, and from bibliographies of relevant articles. We undertook a comprehensive search in PubMed, from 1976, through to 2016, using the terms "ventilator associated pneumonia AND intra-abdominal pressure", "Acute compartment syndrome AND ventilator associated pneumonia", "Intra-abdominal Hypertension AND ventilator associated pneumonia". The search was limited to publications in English. Clinical studies were selected for this review if they reported on the relation between IAP and ventilator associated pneumonia in intensive care unit (ICU) patients. Animal studies were included if they reported on the relation between IAP and ventilator associated pneumonia. All abstracts were reviewed by two independent reviewers (Eleni Papakrivou and Demosthenes Makris). Articles of relevant abstracts were reviewed. All relevant articles were included in this review. After PubMed searches, 195 original articles were selected on abstracts and 40 articles were kept in this review.

\section{VAP and IAH definitions}

VAP diagnosis is challenging $(10,11)$. VAP is usually suspected when a critical care patient develops a new or progressive infiltrate on chest radiograph, leukocytosis, and purulent tracheobronchial secretions (10). In contrast to community-acquired pneumonia, accepted clinical criteria for pneumonia are of limited diagnostic value in definitively establishing the presence of VAP (10). The poor accuracy of clinical criteria for diagnosing VAP should not be surprising considering that purulent tracheobronchial secretions may be invariably present in patients receiving prolonged mechanical ventilation and are seldom caused by pneumonia. In addition, the systemic signs of pneumonia such as fever, tachycardia, and leukocytosis are nonspecific (12). Despite the aforementioned discrepancies the American Thoracic Society (ATS) and the Infectious Diseases Society of America (IDSA) guidelines recommend to use clinical criteria for diagnosis and to obtain lower respiratory tract samples for culture and quantitative or qualitative microbiologic assessment (11).

In contrast to the challenging diagnosis VAP, IAH diagnosis is less difficult to be achieved since it is based only on IAP measurement which can be easily obtained by a urinary catheter (urinary blood pressure is a close approximate of IAP). Significant increases in IAP are seen in a wide variety of conditions commonly encountered in the ICU (13-15) but based on current definition IAH is diagnosed when IAP is $\geq 12 \mathrm{mmHg}$, sustained or repeated, without the characteristic pathophysiology of abdominal compartment syndrome (ACS) (16). ACS describes the combination of increased IAP and organ dysfunction. ACS is defined as the combination of (I) IAH greater than $25 \mathrm{mmHg}$ at $0^{\circ}$; (II) progressive organ dysfunction (urinary output $<0.5 \mathrm{~mL} / \mathrm{kg} / \mathrm{h}$ or $\mathrm{PaO}_{2} / \mathrm{FiO}_{2}<150$ or peak airway pressure $>45 \mathrm{cmH}_{2} \mathrm{O}$ or cardiac index $(\mathrm{CI})<3 \mathrm{~L} / \mathrm{min}-\mathrm{m}^{2}$ despite resuscitation; and (III) improved organ function after decompression.

\section{Abdominal hypertension and the respiratory system}

Pulmonary dysfunction may be the earliest manifestation of IAH (17) and has been already described in the late 1800s (18). Emerson in 1911 hypothesized a reciprocal relationship between IAP and intra-thoracic pressure, with a steady decline in inspired air with respiratory failure and death occurring with IAP above $27-46 \mathrm{cmH}_{2} \mathrm{O}$ in anaesthetized cats and guinea pigs (19). Baggot, an anaesthetist from Dublin, described in 1951 the clinical effects of abdominal wound closure under tension after a dehiscence or "abdominal blow-out". He noted a high mortality with these operations and, referring to earlier investigations, he concluded that death was a result of respiratory dysfunction.

The highest incidence of IAH is observed in patients who have undergone emergency laparotomy for abdominal trauma (20). In this respect, the role of surgical operations is as a risk factor for presenting IAH and it has been pointed out in critical care patients (3). Recent laparoscopic studies demonstrated that even at the relatively low IAP of $10-15 \mathrm{mmHg}$ significant alterations in organ function can be seen $(21,22)$. Increased IAP can displace the diaphragm into the thoracic cavity and is also transmitted across the diaphragm, causing a smaller, but proportionate rise in intra-thoracic pressure 
and compress lung segments (23) while the effect of abdominal distention in chest wall and lung mechanics has been well demonstrated in patients with ARDS (24). Approximately $50 \%$ of IAP is dispersed across the diaphragm and as a result, respiratory system and chest wall compliance, and total lung capacity (TLC) and residual volume are reduced $(3,25)$. In pneumo-peritoneum studies for laparoscopic operations - that can mimic a condition of IAH-increased IAP may decrease functional residual capacity (FRC) and thoraco-pulmonary compliance by $30 \%$ to $50 \%$ or even more in obese patients $(26,27)$. On this ground, IAH is considered responsible of substantial alterations in respiratory system mechanics in critical care patients who present severely elevated IAP, as in ACS (3).

Moreover, multi-organ failure that may be encountered in critical care patients with IAH may aggravate further abdominal distension, alveolar collapse and damage (de-recruitment), worsening gas exchange, and declining compliance Sepsis and acidosis in those patients can lead in excessive fluid resuscitation which in turn, it might lead to bowel edema, capillary leak (28). IAH also reduces perfusion of the nephron while simultaneously resisting glomerular filtration - a combined effect that reduces urine production (6). The result is impaired fluid mobilization and excess of extravascular fluid that may lead to difficult ventilator weaning, increased duration of mechanical ventilation and consequently increased risk for respiratory infections $(29,30)$.

\section{IAH and VAP}

VAP pathophysiology involves several potential mechanisms. There are several points that may serve as a connection between IAH and increased VAP frequency.

First, it has been suggested that IAP may favor VAP because increased abdominal pressure may push the diaphragm upward, impinging on the thoracic cavity. The lungs cannot expand completely and respiratory excursion is thus limited, leading in lung atelectasis; the latter may impair bacterial clearance and may increase the risk for infection (3). However, although such a relationship has been hypothesized on the basis of the atelectasis and of the subsequent lung injury that follows IAH and diaphragmatic changes, no study up to now has been clearly reported it.

Second, in the same way that aspiration of gastrointestinal content may lead to pneumonia, VAP can be induced via micro-aspiration of gastrointestinal content that pass the mechanical barrier of the orotracheal or tracheostomy tube during mechanical ventilation (31). On the other hand, it is well known that IAH leads to reduction of pressure of inferior esophagus sphincter and thus, regression of gastric content (32). Hence, strategies that aimed to reduce microaspirations of gastrointestinal content such as placing patients in a semi-recumbent position or regulating the pressure of the cuff of the orotracheal or tracheostomy tube have shown that may be associated with decreased VAP frequency. Semi-recumbent position was associated with approximately a 3-fold reduction in the risk of VAP, especially during enteral feeding (31). In addition, Nseir et al. suggested that optimal continuous control of the orotracheal cuff pressures or use of conical-cuffed tracheal tube could decrease microaspiration of gastric contents that occur in critically ill patients $(33,34)$. In this respect one might argue that IAH may increase aspiration of gastric content in the lung and in this respect VAP. However, data from studies that assessed directly the above hypothesis are very limited.

In a recent single-center prospective observational cohort study, Papakrivou et al. (35) demonstrated a significant association between VAP and IAH. In that investigation, the authors included a population with increased risk for IAH and demonstrated that 24 patients who presented IAH presented also VAP whereas the incidence of VAP in patients without IAH was $(53.3 \%$ vs. $26.9 \%$ respectively, $\mathrm{P}=0.006$ ). VAP followed IAH in all cases. Notably, patients who presented VAP and IAH presented different microbiology compared to patients who presented VAP without IAH; in particular, Klebsiella species were more frequently isolated in patients with VAP and IAH $(\mathrm{P}=0.0018)$. The authors suggested that one alternative hypothesis for this association between VAP and IAH, besides the aspiration of gastric content, could be the translocation of bacteria from the lung to the bloodstream and/or vice versa. The latter could form a third hypothesis that may explain a relationship between VAP and IAH. Microbial translocation from the intestine during increased IAP may increase the odds for the development of sepsis in distal organs such as the lungs in the patient with bacteremia/sepsis as a result of IAH/ACS due to a consequent loss of barrier function of the alveolo-capillary barrier. In favour of this hypothesis is the effect of IAH on the splanchnic organs that leads to diminished gut perfusion, ischemia, acidosis of the mucosal bed, capillary leak, intestinal edema, increased permeability and translocation of bacteria from the intestinal lumen to regional lymph nodes (36). Notably, local tumor necrosis factor (TNF) 
production is substantially increased in the mesenteric lymph nodes of cirrhotic rats (37) during IAH conditions. Bacterial antigens and endotoxins are potent stimulators of TNF release by mononuclear cells. The increased permeability allows the intestinal flora to translocate via the villi into the lymph and vascular systems. In line with the above, in an experimental megacolon animal model of IAH (9), significant amount of enterobacteroids [Klebciella species, E. Coli, Enterococcus faecalis, Peptostreptococcus (anaerobius, magnus), Pseudomonas aeroginosa] were found in the mesenteric lymph nodes and also in spleen, liver, kidneys and most notably lungs. The study demonstrated also that an increase in IAP in studied animals was associated with lung inflammatory changes and similar bacterial growth in the lungs and in mesenteric lymph nodes. These phenomena were not observed in control animals. These findings may indicate that the responsible underlying mechanism for pneumonia in cases where IAP is highly elevated could be bacterial translocation via the bloodstream, beside the aspiration of gastrointestinal content which is a known pathophysiological mechanism. A reverse phenomenon has been reported in the mechanically ventilated lungs where transpulmonary pressures has been shown to be associated with microbial translocation from the lung into the bloodstream (38-40). Nevertheless, the influence of IAH in inducing bacterial translocation from the intestine in the direction of the human lung is still questionable due to the lack of prospective clinical studies and remains a hypothesis.

\section{Conclusions}

IAH may induce physiologic changes that could favor increased VAP frequency in critical care patients. However, clinical data to support it are very limited. More studies are required to explore the relationship between IAH and VAP and the potential pathophysiological mechanisms.

\section{Acknowledgements}

None.

\section{Footnote}

Conflicts of Interest: The authors have no conflicts of interest to declare.

\section{References}

1. Nseir S, Makris D, Mathieu D, et al. Intensive Care Unit- acquired infection as a side effect of sedation. Crit Care 2010;14:R30.

2. Parmentier-Decrucq E, Nseir S, Makris D, et al. Accuracy of leptin serum level in diagnosing ventilator-associated pneumonia: a case-control study. Minerva Anestesiol 2014;80:39-47.

3. Malbrain ML, De Iaet IE. Intra-abdominal hypertension: evolving concepts. Clin Chest Med 2009;30:45-70.

4. Cheatham ML, Safcsak K, Block EF, et al. Preload assessment in patients with an open abdomen. J Trauma 1999;46:16-22.

5. Quintel M, Pelosi P, Caironi P, et al. An increase of abdominal pressure increases pulmonary edema in oleic acid induced lung injury. Am J Respir Crit Care Med 2004;169:534-41.

6. Sugrue M, Jones F, Janjua KJ, et al. Temporary abdominal closure: A prospective evaluation of its effects on renal and respiratory physiology. J Trauma 1998;45:914-21.

7. Estes RJ, Meduri GU. The pathogenesis of ventilatorassociated pneumonia: Mechanisms of bacterial transcolonization and airway inoculation. Intensive Care Med 1995;21:365-83.

8. De Laet I, Malbrain M, De Waele J. The effect of neuromuscular blockers in patients with IAH. Intensive Care Med 2007;33:1811-14.

9. Papakrivou E, Makris D, Manoulakas E, et al. Intra-Abdominal Hypertension Causes Bacterial Growth in Lungs: An Animal Study. Biomed Res Int 2017;2017:4601348.

10. Makris D, Manoulakas E, Komnos A, et al. Effect of pravastatin on the frequency of ventilator-associated pneumonia and on intensive care unit mortality: openlabel, randomized study. Crit Care Med 2011;39:2440-6.

11. American Thoracic Society, Infectious Diseases Society of America. Guidelines for the management of adults with hospital-acquired, ventilator-associated, and healthcareassociated pneumonia. Am J Respir Crit Care Med 2005;171:388-416.

12. Choudhuri AH. Ventilator-Associated Pneumonia: When to hold the breath? Int J Crit Illn Inj Sci 2013;3:169-74.

13. Kron IL, Harman PK, Nolan SP. The measurement of intra-abdominal pressure as a criterion for abdominal reexploration. Ann Surg 1984;199:28-30.

14. Ridings PC, Bloomfield GL, Blocher CR, et al. Cardiopulmonary effects of raised intra-abdominal pressure before and after intravascular volume expansion. J Trauma 1995;39:1071-5.

15. Eddy V, Nunn C, Morris JA Jr. Abdominal compartment 
syndrome. The Nashville experience. Surg Clin North Am 1997;77:801-12.

16. Kirkpatrick AW, Roberts DJ, De Waele J, et al. Intraabdominal hypertension and the abdominal com partent syndrome: updated consensus definitions and clinical practice guidelines from the World Society of the Abdominal Compartment Syndrome. Intensive Care Med 2013:39;1190-206.

17. Schein M, Wittmann DH, Aprahamian CC, et al. The abdominal compartment syndrome: The physiological and clinical consequences of elevated intra-abdominal pressure. J Am Coll Surg 1995;180:745-53.

18. Mahran GS, Abbas MS. Compartment syndrome: A cornerstone in critical care management. J Anaesthesiol Crit Care 2017;1.

19. Mutoh T, Lamm WJ, Embree LJ, et al. Volume infusion produces abdominal distension, lung compression, and chest wall stiffening in pigs. J Appl Physiol 1992;72:575-82.

20. Ivatury RR, Porter JM, Simon RJ, et al. Intra-abdominal hypertension after life-threatening penetrating abdominal trauma: Prophylaxis, incidence, and clinical relevance to gastric mucosal $\mathrm{pH}$ and abdominal compartment syndrome. J Trauma 1998;44:1016-21; discussion 1021-3.

21. Erice F, Fox GS, Salib YM, et al. Diaphragmatic function before and after laparoscopic cholecystectomy. Anesthesiology 1993;79:966-75; discussion 27A-8A.

22. Sharma RR, Axelsson H, Oberg A, et al. Diaphragmatic activity after laparoscopic cholecystectomy. Anesthesiology 1999;91:406-13.

23. Cullen DJ, Coyle JP, Teplick R, et al. Cardiovascular, pulmonary, and renal effects of massively increased intraabdominal pressure in critically ill patients. Critical Care Medicine 1989;17:118-21.

24. Ranieri VM, Brienza N, Santostasi S, et al. Impairment of lung and chest wall mechanics in patients with acute respiratory distress syndrome: role of abdominal distension. Am J Respir Crit Care Med 1997;156: 1082-91.

25. Kelman GR, Swapp GH, Smith I, et al. Caridac output and arterial blood-gas tension during laparoscopy. Br J Anaesth 1972;44:1155-62.

26. Puri GD, Singh H. Ventilatory effects of laparoscopy under general anaesthesia. Br J Anaesth 1992;68:211-3.

27. Sprung J, Whalley DG, Falcone T, et al. The impact of morbid obesity, pneumoperitoneum, and posture on respiratory system mechanics and oxygenation during laparoscopy. Anesth Analg 2002;94:1345-50.

28. Bongard F, Pianim N, Dubecz S, et al. Adverse consequences of increased intra-abdominal pressure on bowel tissue oxygen. J Trauma 1995;39:519-24; discussion 524-5.

29. Aybar Türkoğlu M, Topeli Iskit A. Ventilator-associated pneumonia caused by high risk microorganisms: a matched case-control study. Tuberk Toraks 2008;56:139-49.

30. Soler Morejón Cde CD, Tamargo Barbeito TO. Effect of mechanical ventilation on intra-abdominal pressure in critically ill patients without other risk factors for abdominal hypertension: an observational multicenter epidemiological study. Ann Intensive Care 2012;2 Suppl 1:S22.

31. Heyland DK, Drover JW, Mac Donald S, et al. Effect of postpyloric feeding on gastroesophageal regurgitation and pulmonary microaspiration: results of a randomized controlled trial. Crit Care Med 2001;29:1495-501.

32. Mittal RK, Fisher M, McCallum RW, et al. Human lower esophageal sphincter pressure response to increased intraabdominal pressure. Am J Physiol 1990;258:G624-30.

33. Jaillette E, Brunin G, Girault C, et al. Impact of tracheal cuff shape on microaspira-tion of gastric contents in intubated critically ill patients: study protocol for a randomized controlled trial. Trials 2015;16:429.

34. Nseir S, Zerimech F, Fournier C, et al. Continuous control of tracheal cuff pressure and microaspiration of gastric contents in critically ill patients. Am J Respir Crit Care Med 2011;184:1041-7.

35. Papakrivou E, Makris D, Manoulakas E, et al. IntraAbdominal Hypertension is a Risk Factor for Increased VAP Incidence: A Prospective Cohort Study in the ICU of a Tertiary Hospital. J Intensive Care Med 2018. [Epub ahead of print].

36. Papavramidis TS, Marinis AD, Pliakos I, et al. Abdominal compartment syndrome - Intra-abdominal hypertension: Defining, diagnosing, and managing. J Emerg Trauma Shock 2011;4:279-91.

37. Genescà J, Marti R, Rolo F, et al. Increased tumour necrosis factor alpha production in mesenteric lymph nodes of cirrhotic patients with ascites. Gut 2003;52;1054-9.

38. Verbrugge SJ, Sorm V, van't Veen A, et al. Lung overinflation without positive end-expiratory pressure promotes bacteremia after experimental Klebsiella pneumoniae inoculation. Intensive Care Med 1998;24:172-7.

39. Tilson MD, Bunke MC, Smith GJ, et al. Quantitative bacteriology and pathology of the lung in experimental 
Pseudomonas pneumonia treated with positive endexpiratory pressure (PEEP). Surgery 1977;82:133-40.

40. Murphy DB, Cregg N, Tremblay L, et al. Adverse

Cite this article as: Papakrivou E, Manoulakas E, Zakynthinos E, Makris D. Is intra-abdominal hypertension a risk factor for ventilator-associated pneumonia? Ann Transl Med 2018;6(21):419. doi: 10.21037/atm.2018.08.27 ventilatory strategy causes pulmonary-to-systemic translocation of endotoxin. Am J Respir Crit Care Med 2000;162:27-33. 